\title{
Patency of conduits in patients who received internal mammary artery, radial artery and saphenous vein grafts
}

Alistair Royse ${ }^{1,2^{*}}$, William Pamment ${ }^{1}$, Zulfayandi Pawanis ${ }^{1,3}$, Sandy Clarke-Errey ${ }^{4}$, David Eccleston ${ }^{5}$, Andrew Ajani ${ }^{5}$, William Wilson ${ }^{5}$, David Canty ${ }^{1,6}$ and Colin Royse ${ }^{1,7}$

\begin{abstract}
Background: Where each patient has all three conduits of internal mammary artery (IMA), saphenous vein graft (SVG) and radial artery (RA), most confounders affecting comparison between conduits can be mitigated. Additionally, since SVG progressively fails over time, restricting patient angiography to the late period only can mitigate against early SVG patency that may have occluded in the late period.
\end{abstract}

Methods: Research protocol driven conventional angiography was performed for patients with at least one of each conduit of IMA, RA and SVG and a minimum of 7 years postoperative. The primary analysis was perfect patency and secondary analysis was overall patency including angiographic evidence of conduit lumen irregularity from conduit atheroma. Multivariable generalized linear mixed model (GLMM) was used. Patency excluded occluded or "string sign" conduits. Perfect patency was present in patent grafts if there was no lumen irregularity.

Results: Fifty patients underwent coronary angiography at overall duration postoperative $13.1 \pm 2.9$, and age $74.3 \pm 7.0$ years. Of 196 anastomoses, IMA 62, RA 77 and SVG 57. Most IMA were to the left anterior descending territory and most RA and SVG were to the circumflex and right coronary territories. Perfect patency RA 92.2\% was not different to IMA 96.8\%, $P=0.309$; and both were significantly better than SVG 17.5\%, $P<0.001$. Patency RA $93.5 \%$ was also not different to IMA 96.8\%, $P=0.169$, and both arterial conduits were significantly higher than SVG $82.5 \%, P=0.029$. Grafting according to coronary territory was not significant for perfect patency, $P=0.997$ and patency $P=0.289$. Coronary stenosis predicted perfect patency for RA only, $P=0.030$ and for patency, RA, $P=0.007$, and SVG, $P=0.032$. When both arterial conduits were combined, perfect patency, $P<0.001$, and patency, $P=0.017$, were superior to SVG. Conclusions: All but one patent internal mammary artery or radial artery grafts had perfect patency and had superior perfect patency and overall patency compared to saphenous vein grafts.

Keywords: (Max 10) patency, Arterial, Saphenous vein, Radial artery, Internal mammary artery

\footnotetext{
* Correspondence: Alistair.Royse@unimelb.edu.au; https://au.linkedin. com/in/alistairroyse; https://www.researchgate.net/profile/Alistair_Royse 'Department of Surgery, The University of Melbourne, PO Box 2135 RMH, Melbourne 3050, Australia

2Department of Cardiothoracic Surgery, The Royal Melbourne Hospital, PO Box 2135, Melbourne, Victoria 3050, Australia

Full list of author information is available at the end of the article
}

C C The Author(s). 2020 Open Access This article is licensed under a Creative Commons Attribution 4.0 International License, which permits use, sharing, adaptation, distribution and reproduction in any medium or format, as long as you give appropriate credit to the original author(s) and the source, provide a link to the Creative Commons licence, and indicate if changes were made. The images or other third party material in this article are included in the article's Creative Commons licence, unless indicated otherwise in a credit line to the material. If material is not included in the article's Creative Commons licence and your intended use is not permitted by statutory regulation or exceeds the permitted use, you will need to obtain permission directly from the copyright holder. To view a copy of this licence, visit http://creativecommons.org/licenses/by/4.0/. The Creative Commons Public Domain Dedication waiver (http://creativecommons.org/publicdomain/zero/1.0/) applies to the data made available in this article, unless otherwise stated in a credit line to the data. 


\section{Background}

Many confounding factors interact to limit direct comparisons between coronary bypass conduits, including coronary target grafting preferences, techniques of reconstruction, medications, individual patient differences or duration postoperatively. For an individual patient, some factors such as age, gender, medications or comorbid diseases should affect conduits equally. For group comparisons of conduits, if each patient has all three of the comparison conduits, this reduces bias compared to some patients not receiving one or more of the conduits.

Our institutional practice had relatively few patients that received all three conduits of internal mammary artery (IMA), radial artery (RA) and saphenous vein (SVG) at the same time, with subsequent experience being predominantly total arterial revascularization $[1,2]$. We wished to examine conduit patency in the late postoperative period without the confounding influence of conduits that may exhibit early patency but could later have occluded, as is known to be the case with SVG where patency at 10 years is $47-64 \%$ [3-9]. Alternatively, arterial conduits may fail in the early period thought to relate to flow competition from the native coronary circulation but with little evidence of progressive failure over the mid or late postoperative periods. Grafting strategies are usually biased where the left IMA (LIMA) is generally anastomosed to the left anterior descending artery (LAD) which has the highest patency; whereas other conduits are used to revascularize the right coronary artery (RCA) which has the lowest patency $[1,10]$. Stenosis or occlusion of the conduit may lead to recurrence of angina, myocardial infarction or heart failure, with reduced survival. We previously found that there was reduced survival even with the use of a single SVG as well as for multiple SVG conduits when compared to total arterial revascularization [11].

The angiographic classification of Fitzgibbon appears relevant only to SVG, since in our observations late angiography of arterial conduits appear to exhibit entirely normal lumen appearance; or are occluded [12]. We hypothesize that if an arterial conduit appears angiographically normal after 10 years postoperative, then it is unlikely to ever fail. Alternatively, if SVG appears irregular, but patent, after 10 years, then it would be expected that some progression of the conduit atherosclerosis would occur which may lead to eventual occlusion of the conduit. We therefore did not use the Fitzgibbon classification.

The presence or absence of symptoms may bias angiographic patency; and survival in the late period could bias results by selecting only long-term survivors. However, within each individual patient, many of the confounding variables can be eliminated if each patient had all three conduits of interest used.
The primary analysis was perfect patency where the presence of conduit lumen irregularity (atheroma) is absent, which may predict ongoing long-term preservation of patency. The secondary analysis was absolute patency where the presence of conduit lumen irregularity may predict possible ongoing loss of conduit patency in the long-term.

\section{Methods}

We conducted a prospective observational angiographic study of patients who had received coronary bypass surgery (CABG) using at least one of each of the three conduits, IMA, RA and SVG, and who were a minimum of 7 years post-operative, with no upper limit to duration postoperative, Fig. 1. Thus, all postoperative angiograms performed in the early and intermediate periods postoperative were excluded. Conventional angiography was used for optimal accuracy of imaging of conduit lumen irregularity. Participants were identified from the Royal Melbourne Hospital institutional database, and mortality was determined by linkage to the national death registry (Australian Institute Health of Welfare). Postmortem examinations are not routinely performed, and no records were available. All angiography was performed at the Royal Melbourne Hospital, receiving research protocol driven conventional angiography between 2012 to 2017.

\section{Ethics approval and consent to participate}

The Melbourne Health human ethics committee approved the study and written informed consent was obtained for

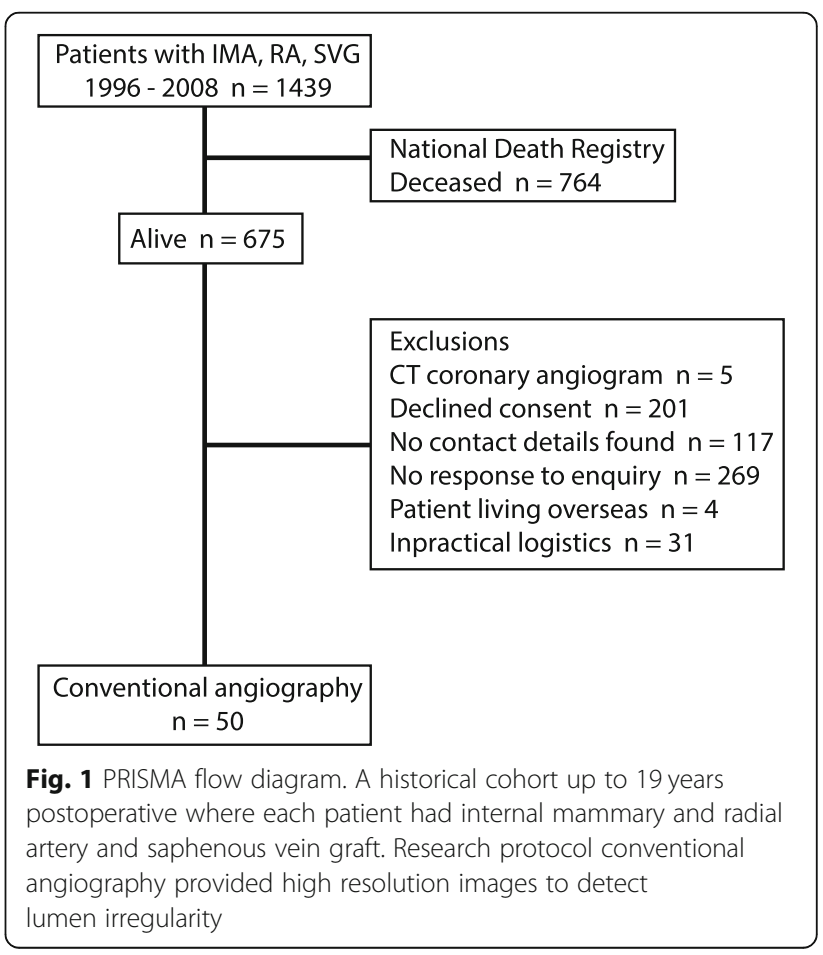


all participants, HREC 2011.164. Living patients were approached if they had received coronary artery bypass surgery where all three types of conduit (IMA, RA and SVG) were used. Participants who refused conventional angiography, had contraindications to angiography, who underwent CT coronary angiogram, or had angiography for clinical indications, were excluded, Figs. 1 and 2. The cardiologist performing the angiogram and a researcher were the observers and neither were blinded to the grafting strategy. Following angiography, the report was distributed to the patient's general practitioner and usual treating cardiologist; and the results were discussed directly with the patient at the time of the angiogram. At no time was a therapeutic intervention undertaken or advised at the same time as the research angiogram. The selection of the conduits and the grafting targets were at the sole discretion of the surgeon. However, during the study time frame, it was usual practice at this institution to graft coronary targets with lesion severity of $\geq 50 \%$; and precise degrees of coronary stenosis and related patency are presented in the Supplement, Table S1. There were no details provided in the operative notes to explain the selected strategy by the surgeon. Institutional policy was for all patients to be discharged from hospital with a lipid lowering medication and low dose aspirin.

\section{Conduit harvest}

During this study time frame, the LIMA was harvested in a pedicled (non-skeletonized) manner, the RA harvested with an open technique and side branches divided with electrocautery between metal clips and with the use of topical and intraluminal $1 \%$ papaverine solution and no intravenous systemic vasodilators; and SVG harvested using an open technique with side branches divided between fine ties or metal clips without additional fat being included adjacent to the vein and gentle dilation of the vein using saline.

\section{Primary analysis: assessment of perfect patency (absence of conduit lumen irregularity)}

The lumen was examined for evidence of irregularity in the same manner as for the native coronary artery angiography. Any irregularity (even a minor irregularity) was classified as irregular. Absence of irregularity was classified as normal. The term "Perfectly Patent", refers to a conduit being both patent and having a normal lumen angiographic appearance.

\section{Secondary analysis: assessment of patency (patent even in presence of conduit lumen irregularity)}

Conduits were classified as patent or occluded. Arterial grafts considered to have a "string sign" (a diffusely narrowed conduit that failed to fill the native coronary artery via the graft injection), were classified as occluded. Sequential anastomoses were considered as separate grafts.

\section{Statistics}

For continuous variables, the means and standard deviations were provided. In order to adjust for patient level effects and other risk factors, generalised linear mixed model analysis (GLMM) was used, with patency and perfect patency as outcomes, patient as the random effect, and type of conduit as the key predictor. The patient level variables included in these models were duration to the research angiogram postoperatively, diabetes, hypertension, hypercholesterolaemia and atrial fibrillation, and graft level variables of preoperative native coronary stenosis and coronary territory. The patient random effect on patency $(v a r=0.60$, se $=0.92)$ and perfect patency (var $=0.22$, se $=0.74$ ) was small.

A sensitivity test was performed where specific exclusions were made including the RA graft known to be calcified at implantation, the patient describing post angiogram symptoms, or for angiograms performed > 10 years postoperative; or patients from all three of these scenarios being excluded, see Supplementary Materials; and all were consistent with the main analyses.

\section{Results}

Of an original cohort of 1439 patients, 675 remained alive at the times of contact, and a further 627 were excluded, with the majority unable to be located or declined consent, Fig. 1. Fifty patients had angiography
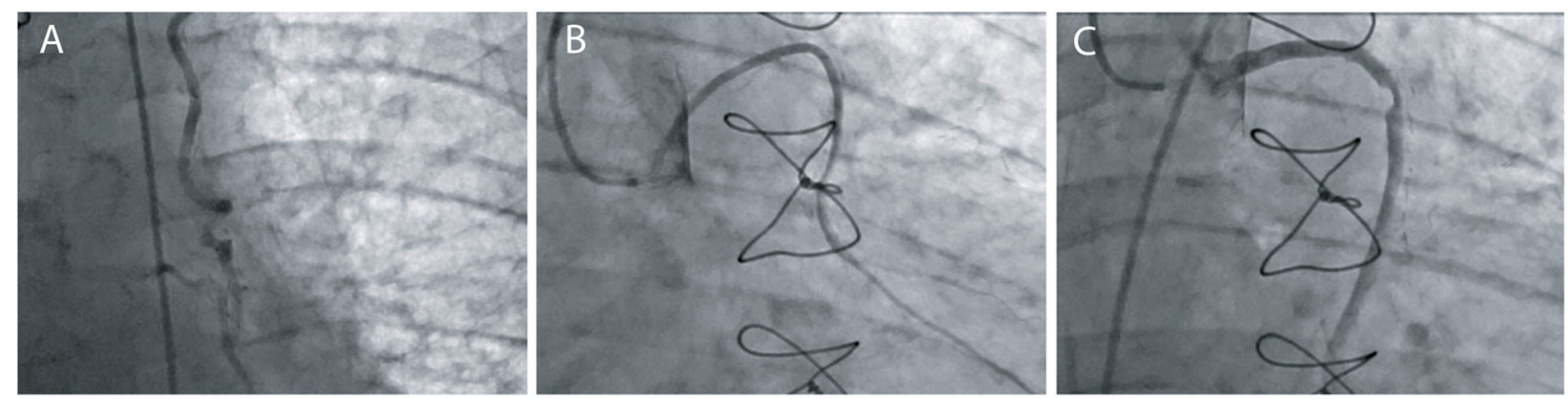

Fig. 2 Conduit angiogram examples. a, left internal mammary artery, b, radial artery, c, saphenous vein 
at age $74.3 \pm 7.0$ (range 57-90) years. Research protocol driven angiography was performed at $13.1 \pm 2.9$ (range 7-19) years postoperative. Patients received $3.9 \pm 1.0$ (range 3-6) anastomoses. Demographic details are listed in Table 1. Six patients had previous postoperative angiography at least 7 years prior to their current research angiogram; one of whom had a drug eluting stent placed to a diseased SVG at 9 years postoperatively; and at research angiography at 16 years postoperatively ( 7 years post stent), this graft was occluded. One patient denied chest pain symptoms at the time or recruitment; however, after the angiogram the patient admitted to mild atypical chest pain symptoms; and had patent grafts.

\section{Distribution of anastomoses}

There were 196 anastomoses which were relatively evenly distributed between conduits IMA $n=62$, RA $n=$ 77 and SVG $n=57$. The distribution of grafts was biased by IMA predominantly being grafted to the LAD territory, with RA and SVG predominantly elsewhere, $P<$ 0.001 , Tables 2 and 3 . Within the LAD territory, RA and SVG were predominantly grafted to the diagonal arteries. Details of anastomosis distribution according to conduit, coronary stenosis and coronary territory are listed in Supplemental Table S1. Although there were differences shown in the raw data within the comparisons of patency according to the coronary territory, after an adjustment of the conduit to the model, there were no influences on perfect patency, $P=0.289$ or patency, $P=$ 0.997 , Table 4 . Sequential grafting was mostly with arterial conduits and composite Y-grafting predominantly occurred with RA, Table 2.

\section{Global predictors}

Conduit type was the only significant predictor for perfect patency, $P<0.001$. Preoperative native coronary stenosis OR $1.07,95 \% \mathrm{CI}(1.02,1.11), P=0.001$; duration to the

Table 1 Demographic details $n=50$

\begin{tabular}{ll}
\hline Variable & $\boldsymbol{n}(\%)$ \\
\hline Male & $48(96)$ \\
Symptoms & $1(2)$ \\
Dirrent smoker & $2(4)$ \\
Hypertension & $21(42)$ \\
Hypercholesterolemia & $37(74)$ \\
Family history of IHD & $29(58)$ \\
Dialysis & $1(2)$ \\
Chronic lung disease & $2(4)$ \\
Atrial fibrillation & $1(2)$ \\
Coronary stent pre-research angiogram & $8(16)$ \\
\hline
\end{tabular}

IHD ischaemic heart disease
Table 2 Distribution of anastomoses

\begin{tabular}{|c|c|c|c|c|}
\hline & $\begin{array}{l}\text { IMA } \\
n(\%)\end{array}$ & $\begin{array}{l}\text { RA } \\
\boldsymbol{n}(\%)\end{array}$ & $\begin{array}{l}\text { SVG } \\
n(\%)\end{array}$ & $\begin{array}{l}\text { Total } \\
\boldsymbol{n}(\%)\end{array}$ \\
\hline \multicolumn{5}{|c|}{ Coronary Territory } \\
\hline LAD & 57 & 8 & 6 & $71(36)$ \\
\hline$C x$ & 4 & 50 & 18 & $72(37)$ \\
\hline $\mathrm{RCA}$ & 1 & 19 & 33 & $53(27)$ \\
\hline Total & $62(32)$ & 77 (39) & $57(29)$ & $196(100)$ \\
\hline \multicolumn{5}{|l|}{ Anastomosis } \\
\hline End-Side & 53 & 67 & 56 & 176 \\
\hline Sequential & 9 & 10 & 1 & 20 \\
\hline \multicolumn{5}{|l|}{ Graft Origin } \\
\hline Aorta & 6 & 70 & 56 & 132 \\
\hline Pedicled & 56 & 0 & 0 & 56 \\
\hline Y graft & 0 & 7 & 1 & 8 \\
\hline
\end{tabular}

LAD left anterior descending artery territory, IMA internal mammary artery, $R A$ radial artery, SVG saphenous vein graft, pedicled, origin from the subclavian artery, Y graft, composite graft between two conduits

research angiogram OR $0.69,95 \mathrm{CI}(0.51,0.91), P=0.010$, and conduit type $P=0.049$, were predictors of patency.

\section{Conduit analysis}

Perfect patency was $96.8 \%$ for IMA, $92.2 \%$ for RA, and $17.5 \%$ for SVG indicating that the majority of patent SVG had atheroma present and the arterial grafts were normal. The IMA perfect patency was not significantly different to the RA (absolute difference 4.6\%, $P=0.265$ ) but was higher than SVG (absolute difference $79.3 \%, P<0.001)$. Similarly, RA perfect patency was significantly higher than SVG (absolute difference $74.7 \%, P<0.001$ ).

Table 3 Distribution of conduits according to coronary branch target

\begin{tabular}{lllll}
\hline Coronary branch & IMA & RA & SVG & Total \\
\hline LAD & 46 & 1 & 1 & 48 \\
D1 & 10 & 5 & 5 & 20 \\
D2 & 1 & 2 & 0 & 3 \\
Intermediate & 1 & 5 & 3 & 9 \\
M1 & 2 & 21 & 9 & 32 \\
M2 & 0 & 19 & 5 & 24 \\
M3 & 1 & 5 & 1 & 7 \\
RCA & 0 & 1 & 1 & 2 \\
PDA & 1 & 14 & 26 & 41 \\
LVBr & 0 & 4 & 6 & 10 \\
Total & 62 & 77 & 57 & 196 \\
\hline
\end{tabular}

Intermediate artery was grouped with the circumflex territory IMA internal mammary artery, RA radial artery, SVG saphenous vein graft, LAD left anterior descending artery, D1-2 diagonal arteries, M1-3 marginal arteries, $R C A$ right coronary artery, $P D A$ posterior descending artery, $L V B r$ left ventricular branch artery 
Table 4 Multivariable comparison of patency according to coronary territory

\begin{tabular}{lllll}
\hline Comparison & $\begin{array}{l}\text { Perfect Patency } \\
\boldsymbol{n}(\%)\end{array}$ & P (GLMM) & $\begin{array}{l}\text { Patency } \\
\boldsymbol{n}(\%)\end{array}$ & P (GLMM) \\
\hline LAD, CX, RCA & & 0.997 & & 0.289 \\
LAD & $63 / 71(88.7)$ & 0.940 & $66 / 71(93.0)$ & 0.277 \\
CX & $53 / 72(73.6)$ & & $67 / 72(93.1)$ & \\
LAD & $63 / 71(88.7)$ & 0.941 & $66 / 71(93.0)$ & 0.901 \\
RCA & 25/53(47.2) & & $46 / 53(86.8)$ & \\
CX & $53 / 72(73.6)$ & 0.997 & $67 / 72(93.1)$ & 0.136 \\
RCA & 25/53(47.2) & & $46 / 53(86.8)$ & \\
\hline
\end{tabular}

$P$ (GLMM) $P$ value adjusted for patient level effects and other risk factors, GLMM generalised linear mixed model analysis (see Methods for variables), $L A D$ left anterior descending artery, $C x$ circumflex artery, $R C A$ right coronary artery, univariable analysis, see Supplement Table S7

The incidence of patency and perfect patency for all grafts is shown in Table 5, Supplemental Tables S1 and S2 and Fig. 3. All patent IMA were perfectly patent, and only one patent RA was not perfectly patent. Patency for IMA was $96.8 \%$, RA was $93.5 \%$, and SVG was $82.5 \%$. The IMA patency was not significantly different to the RA (absolute difference $3.3 \%, P=0.146$ ) but was higher than SVG (absolute difference $14.3 \%, P=0.016)$. The RA patency was higher than for SVG (absolute difference 11\%), however but was the difference was not significant $(P=0.170)$. When combining all arterial grafts versus SVG, both perfect patency and patency were higher for arterial grafts (perfect patency $94.2 \%$ vs. $17.5 \%, P<0.001$ and patency $95 \%$ vs. $82.5 \%, P=0.033)$.

In the case of the single radial artery graft that did exhibit lumen irregularity, the operative report detailed significant calcification of this right RA at the time of the surgery and at late angiography, this RA conduit remained patent with an irregular lumen, Fig. 4, Supplemental Figure S1; but it was not possible to determine if

Table 5 Multivariate comparison of patency according to conduit

\begin{tabular}{lllll}
\hline Comparison & $\begin{array}{l}\text { Perfect patency } \\
\boldsymbol{n}(\%)\end{array}$ & $\begin{array}{l}\text { P } \\
(\text { GLMM })\end{array}$ & $\begin{array}{l}\text { Patency } \\
\boldsymbol{n}(\%)\end{array}$ & $\begin{array}{l}\text { P } \\
\text { (GLMM) }\end{array}$ \\
\hline IMA, RA, SVG & & $<0.001$ & & 0.049 \\
IMA vs. & $60 / 62(96.8)$ & 0.309 & $60 / 62(96.8)$ & 0.169 \\
RA & $71 / 77(92.2)$ & & $72 / 77(93.5)$ & \\
IMA vs. & $60 / 62(96.8)$ & $<0.001$ & $60 / 62(96.8)$ & 0.021 \\
SVG & $10 / 57(17.5)$ & & $47 / 57(82.5)$ & \\
RA vs. & $71 / 77(92.2)$ & $<0.001$ & $72 / 77(93.5)$ & 0.175 \\
SVG & $10 / 57(17.5)$ & & $47 / 57(82.5)$ & \\
Arterial, SVG & & $<0.001$ & & 0.037 \\
Arterial vs. & $131 / 139(94.2)$ & $<0.001$ & $132 / 139(95.0)$ & 0.037 \\
SVG & $10 / 57(17.5)$ & & $47 / 57(82.5)$ & \\
\hline P (GLMM) P value & & & & \\
\hline
\end{tabular}

$P$ (GLMM) $P$ value adjusted for patient level effects and other risk factors, GLMM generalised linear mixed model analysis (see Methods for variables), IMA internal mammary artery, RA radial artery, SVG saphenous vein graft, see Supplementary Materials for sensitivity testing, univariable analysis, see Supplement Table S2

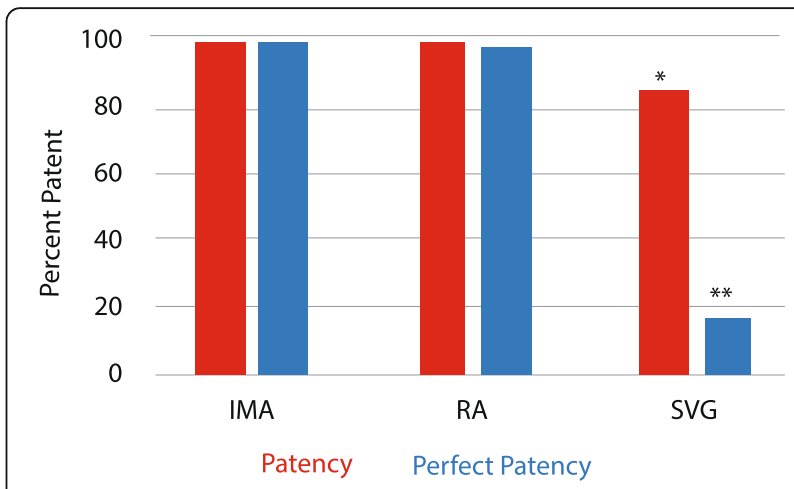

Fig. 3 Conduit patency and perfect patency (GLMM) $n=196$ anastomoses. Arterial conduit patency was high, not different from each other despite differing coronary territory grafting and all but one was also perfectly patent. However, vein graft had lower patency and very low perfect patency with progressive decline over time expected. GLMM, generalized linear mixed model analysis, IMA, internal mammary artery, RA, radial artery, SVG, saphenous vein graft, ${ }^{*}, P=0.021$ SVG vs. IMA, ${ }^{*}, P<0.001$ SVG vs. IMA or RA

there had been any progression of calcification or atheroma in the postoperative period. Additional sensitivity analyses were therefore conducted, see Supplement Tables S3, S4, S5 and S6, which did not alter the findings.

\section{Effect of preoperative coronary artery stenosis}

The overall effect of the degree of coronary stenosis present at the time of surgery was not significant for perfect patency, $P=0.317$; but was significant for patency, $P<0.001$, Table 6 . RA and SVG were significantly impacted for patency, but IMA was not. The full distribution of coronary stenosis with conduit type and territory is listed in Supplement Table S1 and conduit distribution according to stenosis grouped ranges Supplement Tables S7 and S8.

Patients in whom all three conduits were considered perfectly patent $(n=6)$, there were no statistically significant differences with the remainder of patients $(n=43)$, Table 7.

\section{Discussion}

\section{High perfect patency in arterial conduits}

The most important finding is that arterial conduits that were patent, appeared normal in the late period postoperatively. This is different to SVG which rarely appears normal in the late period, with most $(82.5 \%$ in this series) having some lumen irregularity consistent with conduit wall atheroma. Although, it is well known for patent LIMA to appear normal irrespective of the duration postoperatively, it has generally been assumed that this would not be true for RA. These data find that when patent, both arterial conduits appear angiographically normal (atherosclerosis-free) in the late period. The conclusion to be drawn is that a diseased conduit such as 


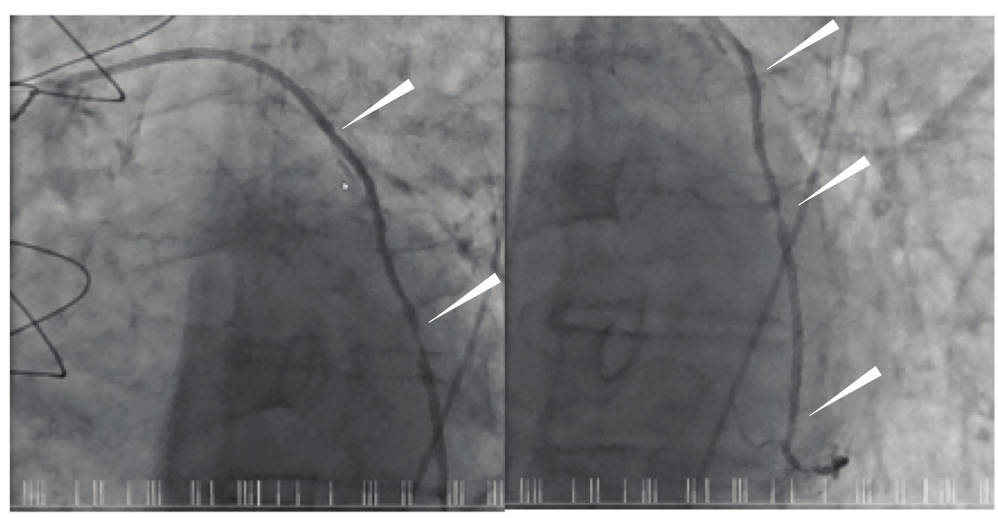

Fig. 4 The only non-perfectly patent arterial conduit. The only patent, but diseased arterial conduit was a radial artery to second marginal, illustrated by arrows. However, it was noted to be diseased at the time of surgery 11 years prior and it is not known if there has been any progression postoperatively. The native coronary artery has severe disease and was noted to be diseased at the time of surgery. https://s3. amazonaws.com/igraft/3Vangio/PreopDiseasedRA.mp4. From the operation report: "The left radial artery was exposed but on harvesting was found to be extensively calcified and not useable. The right radial artery was harvested; this was a $2.2 \mathrm{~mm}$ artery with at least moderate medial wall calcification"

SVG may be expected to have ongoing progressive atheroma formation which ultimately, may cause graft failure by way of hemodynamically significant stenosis or occlusion; whereas the normal arterial conduits may be expected to remain normal indefinitely.

In the single case of a diseased RA, it was noted that there was significant disease present at the time of surgical implantation 11 years prior to angiography, Fig. 4, Supplemental Figure S1. This conduit did not occlude in the interim which was surprising, and the expectation of what may have occurred with SVG. What is not clear is if there was any progression in the severity of the RA disease postoperatively, or alternatively, if the degree of disease remained stable.

\section{High patency of arterial conduits}

By selecting patients in the late period only, we have reduced the probability that a patent SVG would have been recorded in the early or intermediate period, that could have later occluded in the late period. Thus, all patients were from the late period postoperatively. Both conduits had superior perfect patency and patency

Table 6 Comparison of conduit patency according to the influence of preoperative coronary stenosis

\begin{tabular}{lll}
\hline Variable & Perfect patency P (GLMM) & Patency P (GLMM) \\
\hline IMA, RA, SVG & 0.317 & $<0.001$ \\
IMA & 0.204 & 0.133 \\
RA & 0.030 & 0.007 \\
SVG & 0.275 & 0.032 \\
\hline
\end{tabular}

$P$ (GLMM) $P$ value adjusted for patient level effects and other risk factors, GLMM generalised linear mixed model analysis (see Methods for variables), IMA internal mammary artery, RA radial artery, SVG saphenous vein graft compared to SVG at a mean of more than 10 years postoperative, Table 5, Fig. 3.

\section{Coronary territory}

The similarity of IMA and RA patencies despite clear grafting preferences for IMA to the LAD territory and RA for the non-LAD territory, is a novel finding. We attempted to reduce bias by the use of research protocol driven rather than symptom indicated angiography and

Table 7 COPD, chronic obstructive pulmonary disease, AF, atrial fibrillation, $\mathrm{PCl}$, percutaneous coronary intervention, Redo CAGS, reoperation coronary artery bypass surgery

\begin{tabular}{llll}
\hline Variable & $\begin{array}{l}\text { Patent + normal all } 3 \\
\text { conduits }(\boldsymbol{n}=6)\end{array}$ & Other $(\boldsymbol{n}=43)$ & $\boldsymbol{P}$ value \\
\hline Age & $58.8 \pm 9$ & $61.2 \pm 7.2$ & 0.455 \\
Gender & $6(100)$ & $41(95.3)$ & 1 \\
Preoperative factors & & $2(4.7)$ & 1 \\
Current Smoker & 0 & $16(37.2)$ & 0.21 \\
Diabetes & $4(66.7)$ & $31(72.1)$ & 1 \\
Hypertension & $5(83.3)$ & $1(2.4)$ & 1 \\
Family History & 0 & $24(55.8)$ & 0.688 \\
Cholesterol & $4(66.7)$ & $1(2.3)$ & 1 \\
Dialysis & 0 & $3(7)$ & 1 \\
COPD & 0 & $6(14)$ & 1 \\
AF & $1(16.7)$ & $1(2.3)$ & 1 \\
Postoperative factors & & 0 & 1 \\
Angina & 0 & $2(4.7)$ & \\
Myocardial Infarction & 0 & 0 & \\
PCl & 0 & 0 & \\
Redo CAGS & 0 & & \\
\hline
\end{tabular}


the presence of all three conduits within each patient allowing for elimination of many patient and medication factors between conduits within each individual patient. However, the preferences in grafting strategy did not alter by our approach. Yet, we found no differences according to coronary territory, Table 4 . This was surprising as there are many studies that demonstrate highest patency in the LAD and lowest patency in the RCA territories $[1,10]$. These data could potentially challenge some conventional wisdoms. The first, LIMA-LAD being unique in some way, is based on the historical considerations of Loop in 1986 [13] . Their analysis considered LIMA (as the only arterial conduit), being applied to the LAD (the only coronary target for LIMA); and all other grafts were SVG. An alternative interpretation of their data is that they observed the survival impact of one arterial graft compared to the exclusive use of venous grafts. Our data suggest an alternative to the conventional view - that all arterial grafts, if they remain patent in the early period - may have long term, perfect patency. With this hypothesis, the similarity of perfect patency according to coronary territory is explained.

RA and SVG were both grafted predominantly to the non-LAD territories and RA perfect patency was higher than SVG, $P<0.001$, see Supplemental Table S1. Patency was not significant despite an $11 \%$ absolute difference, and it is considered likely that a Type II statistical error due to the small sample size was present. Combining both arterial conduits still maintained superiority over SVG, Table 5.

The patency of SVG of $82.5 \%$, was higher than expected from the literature (47-64\%) [3-7], for this time period post operatively. The higher patency may have reflected survivor bias, which may have been unrelated to conduit selection. Alternatively, a higher proportional of grafts being arterial conduits being used 139/196 (71\%), and with a lower failure rate may have led to improved survival. However, the majority of SVG showed evidence of atherosclerosis with only $17.5 \%$ being considered normal. The observed difference between arterial and venous conduits were not due to poor results in the venous group.

\section{Coronary stenosis}

The effect of coronary stenosis was significant for RA for both perfect patency and for patency; and was significant for patency for SVG, Table 6. These findings are expected; although the interaction with coronary territory is a confounder. Examining the individual anastomoses by coronary territory, conduit and degree of coronary stenosis, for anastomoses to coronary stenosis of $<80 \%$ were mostly patent for RA and SVG; in contrast to the conventional view that most or all of such anastomoses would fail due to competitive flow, Table 4.

\section{Study implications}

Arterial conduits that did not fail in the early period, showed no evidence of progressive atheroma and so theoretically may never fail; whereas most SVG that do not fail in the early period can still be expected to develop conduit atheroma over time which would be expected to be progressive. We have previously reported a survival advantage to total arterial revascularisation compered to any use of SVG [11]. The absence of progressive disease in arterial grafts may be the mechanism whereby better long-term survival occurs. Further, there may be relatively little difference between IMA and RA grafts, and that arterial grafts should be considered as equivalent grafts from this study, noting considerable bias for IMA use to the LAD territory. We have reported that the LIMA-RA-Y graft configuration provides the same survival advantage as other total arterial revascularisation configurations, and is superior to any use of SVG in the late period after surgery with low donor site morbidity $[14,15]$.

\section{Study strengths and limitations}

The key strength of this study is that each patient had at least one of the three conduits of interest. Consequently, patients acted as their own controls, with identical patient factors affecting all of the conduits equally; other than for the influence of the specific coronary target to which the conduit was grafted. The second key strength was that the cohort was within the "late" period postoperative period. This mitigates the common failing of many series whereby some of the cohort lie within the "early" or "intermediate" period during which time there could be higher SVG patency that could potentially decrease, as more SVG occlude prior to the "late" period of more than 10 years. Use of conventional angiography allowed optimal examination of the lumen for irregularity as a marker of conduit atherosclerosis; and this enhanced the validity of this part of the analysis in comparison to CT coronary angiography, which was considered to be less accurate.

The key weaknesses of the study are that it is a nonrandomised, observational study, included only survivors (which may positively bias patency to be higher than for non-survivors), and excluded patients who had angiography for clinical indications (i.e. restricted to research protocol consenting patients). Whilst the patient, environment and medication factors would be identical between conduits for each individual patient, differences could exist between patients and strict control of all such variables was not possible. Additionally, there was a predominant bias for use of the IMA to the LAD territory; and for the other two conduits for the non-LAD territory and this may potentially negatively bias the patency of SVG and RA compared to IMA. Finally, the sample size was 
relatively small as many late term survivors were very old and did not want any further investigation.

\section{Conclusion}

All but one patent internal mammary artery or radial artery grafts had perfect patency and had superior perfect patency and overall patency compared to saphenous vein grafts.

\section{Supplementary information}

Supplementary information accompanies this paper at https://doi.org/10. 1186/s12872-020-01433-0.

\begin{abstract}
Additional file 1: Table S1. Degree of coronary stenosis at the time of surgery according to conduit type and coronary territory. Table S2. Multivariate comparison of patency according to conduit. Table S3. Comparison of patency rates excluding patient with known preoperative radial artery disease $n=192$ anastomoses. Table S4. Comparison of patency rates excluding patient with angiogram at 7 years $n=193$ anastomoses. Table S5. Comparison of patency rates excluding patient who did not reveal presence of symptoms until after angiography $n=$ 191 anastomoses. Table S6. comparison of patency rates with combined exclusion of preoperative RA disease and patient with angiography at 7 years and patient who declared symptoms after angiography $n=184$ anastomoses. Table S7. Multivariable and univariable comparison of patency according to coronary territory. Table S8. Distribution of grafts by coronary territory and categories of coronary stenosis. Figure S1. The only radial artery with irregular lumen 10.6 years postoperative (which was calcified at the time of surgery).
\end{abstract}

\section{Abbreviations}

LIMA: Left internal mammary artery; IMA: Internal mammary artery; RA: Radia artery; SVG: Saphenous vein graft; LIMA-RA-Y: Composite graft where RA is sutured to the side of LIMA; Patent: Conduit which conducts blood to the target coronary artery; Perfectly patent: Conduit where conventional angiographic lumen appearance has no irregularity (normal); CABG: Coronary artery bypass surgery; GLMM: Generalized linear mixed model analysis

\section{Acknowledgements}

The authors wish to acknowledge the work of Lynda Tivendale for her coordination of the project.

\section{Authors' contributions}

All authors AR, WP, ZP, SCE, DE, AA, WW, DC and CR contributed to the manuscript and authors that conducted analyses were AR, WP, DC and ZP. $A R$ and $C R$ were responsible for the study design.

\section{Funding}

NIL

\section{Availability of data and materials}

The datasets used and/or analysed during the current study are available from the corresponding author on reasonable request.

\section{Ethics approval and consent to participate}

The Melbourne Health human ethics committee approved the study and full informed written consent was obtained for all participants, HREC 2011.164.

\section{Consent for publication}

Not applicable.

\section{Competing interests}

The authors declare that they have no competing interests.

\section{Author details}

'Department of Surgery, The University of Melbourne, PO Box 2135 RMH, Melbourne 3050, Australia. ${ }^{2}$ Department of Cardiothoracic Surgery, The Royal Melbourne Hospital, PO Box 2135, Melbourne, Victoria 3050, Australia.
${ }^{3}$ Universitas Airlangga Hospital, Universitas Airlangga, Surabaya, Indonesia. ${ }^{4}$ Statistical Consulting Centre, The University of Melbourne, 139 Barry St, Parkville 3010, Australia. ${ }^{5}$ Department of Medicine and Cardiology, Royal Melbourne Hospital, The University of Melbourne, Melbourne, Australia. ${ }^{6}$ Department of Medicine, Monash University, Clayton, Australia. ${ }^{7}$ Department of Anaesthesia and Pain Management, The Royal Melbourne Hospital, Melbourne, Australia.

Received: 17 June 2019 Accepted: 13 March 2020

Published online: 24 March 2020

\section{References}

1. Royse AG, Royse CF, Tatoulis J, Grigg LE, Shah P, Hunt D, Better N, Marasco SF. Postoperative radial artery angiography for coronary artery bypass surgery. Eur J Cardiothorac Surg. 2000;17(3):294-304.

2. Royse AG, Royse CF, Tatoulis J. Total arterial coronary revascularization and factors influencing in- hospital mortality. Eur J Cardiothorac Surg. 1999;16(5):499-505.

3. Goldman S, Zadina K, Moritz T, Ovitt T, Sethi G, Copeland JG, Thottapurathu L, Krasnicka B, Ellis N, Anderson RJ, et al. Long-term patency of saphenous vein and left internal mammary artery grafts after coronary artery bypass surgery: results from a Department of Veterans Affairs Cooperative Study. J Am Coll Cardiol. 2004:44(11):2149-56.

4. Samano N, Geijer H, Liden M, Fremes S, Bodin L, Souza D. The no-touch saphenous vein for coronary artery bypass grafting maintains a patency, after 16 years, comparable to the left internal thoracic artery: a randomized trial. J Thorac Cardiovasc Surg. 2015;150(4):880-8.

5. Sabik JF 3rd, Lytle BW, Blackstone EH, Houghtaling PL, Cosgrove DM. Comparison of saphenous vein and internal thoracic artery graft patency by coronary system. Ann Thorac Surg. 2005;79(2):544-51 discussion 544-551.

6. Tranbaugh RF, Dimitrova KR, Friedmann P, Geller CM, Harris LJ, Stelzer $P$, Cohen BM, Ko W, DeCastro H, Lucido D. Coronary artery bypass grafting using the radial artery: clinical outcomes, patency, and need for reintervention. Circulation. 2012:126(11 suppl 1):S170-5.

7. Tranbaugh RF, Dimitrova KR, Friedmann P, Geller CM, Harris LJ, Stelzer $P$, Cohen B, Hoffman DM. Radial artery conduits improve long-term survival after coronary artery bypass grafting. Ann Thorac Surg. 2010;90(4):1165-72.

8. Sabik JF 3rd. Understanding saphenous vein graft patency. Circulation. 2011; 124(3):273-5.

9. Mehta RH, Ferguson TB, Lopes RD, Hafley GE, Mack MJ, Kouchoukos NT, Gibson CM, Harrington RA, Califf RM, Peterson ED, et al. Saphenous vein grafts with multiple versus single distal targets in patients undergoing coronary artery bypass surgery: one-year graft failure and five-year outcomes from the Project of Ex-Vivo Vein Graft Engineering via Transfection (PREVENT) IV trial. Circulation. 2011;124(3):280-8.

10. Achouh $P$, Isselmou KO, Boutekadjirt R, D'Alessandro C, Pagny JY, Fouquet $R$, Fabiani JN, Acar C. Reappraisal of a 20-year experience with the radial artery as a conduit for coronary bypass grafting. Eur J Cardiothorac Surg. 2012;41(1):87-92.

11. Royse A, Pawanis Z, Canty D, Ou-Young J, Eccleston D, Ajani A, Reid CM, Bellomo R, Royse $C$. The effect on survival from the use of a saphenous vein graft during coronary bypass surgery: a large cohort study. Eur J Cardiothorac Surg. 2018;54(6):1093-100.

12. FitzGibbon GM, Burton JR, Leach AJ. Coronary bypass graft fate: angiographic grading of 1400 consecutive grafts early after operation and of 1132 after one year. Circulation. 1978;57(6):1070-4.

13. Loop FD, Lytle BW, Cosgrove DM, Stewart RW, Goormastic M, Williams GW, Golding LA, Gill CC, Taylor PC, Sheldon WC, et al. Influence of the internalmammary-artery graft on 10-year survival and other cardiac events. N Engl J Med. 1986;314(1):1-6.

14. Royse A, Brennan A, Ou-Young J, Pawanis Z, Canty D, Royse C. 21-year survival of left internal mammary artery-radial artery-Y graft. J Am Coll Cardiol. 2018;72(12):1334-40.

15. Royse AG, O'Donnell MJ, Mocioaca L, Sharma V, Ou-Young JC, Canty DJ, Royse CF. Does radial artery harvest for coronary surgery compromise forearm blood flow to 22 years post-operative? J Am Coll Cardiol. 2018;72(16):1981-2.

\section{Publisher's Note}

Springer Nature remains neutral with regard to jurisdictional claims in published maps and institutional affiliations. 